OPEN ACCESS

Edited by:

Clement Adebajo Meseko, National Veterinary Research Institute (NVRI), Nigeria

Reviewed by:

Chen Zhao,

Fudan University, China Ricardo Soto-Rifo,

University of Chile, Chile

*Correspondence: Ajit Chande ajitg@iiserb.ac.in

${ }^{\dagger}$ These authors have contributed equally to this work

Specialty section: This article was submitted to Virus and Host, a section of the journal Frontiers in Cellular and Infection Microbiology

Received: 03 February 2021 Accepted: 08 April 2021 Published: 23 April 2021

Citation: Mishra T, Sreepadmanabh M, Ramdas $P$, Sahu AK, Kumar A and

Chande A (2021) SARS CoV-2

Nucleoprotein Enhances the Infectivity of Lentiviral Spike Particles. Front. Cell. Infect. Microbiol. 11:663688. doi: 10.3389/fcimb.2021.663688

\section{SARS CoV-2 Nucleoprotein Enhances the Infectivity of Lentiviral Spike Particles}

\author{
Tarun Mishra ${ }^{1 \dagger}$, M. Sreepadmanabh ${ }^{1 \dagger}$, Pavitra Ramdas ${ }^{1}$, Amit Kumar Sahu ${ }^{1}$, Atul Kumar ${ }^{2}$ \\ and Ajit Chande ${ }^{1 *}$

\begin{abstract}
${ }^{1}$ Molecular Virology Laboratory, Department of Biological Sciences, Indian Institute of Science Education and Research, Bhopal, India, ${ }^{2}$ Structural Biology Laboratory, Department of Biological Sciences, Indian Institute of Science Education and Research, Bhopal, India
\end{abstract}

The establishment of SARS CoV-2 spike-pseudotyped lentiviral (LV) systems has enabled the rapid identification of entry inhibitors and neutralizing agents, alongside allowing for the study of this emerging pathogen in BSL-2 level facilities. While such frameworks recapitulate the cellular entry process in ACE2+ cells, they are largely unable to factor in supplemental contributions by other SARS CoV-2 genes. To address this, we performed an unbiased ORF screen and identified the nucleoprotein $(\mathrm{N})$ as a potent enhancer of spike-pseudotyped LV particle infectivity. We further demonstrate that the spike protein is better enriched in virions when the particles are produced in the presence of $\mathrm{N}$ protein. This enrichment of spike renders LV particles more infectious as well as less vulnerable to the neutralizing effects of a human IgG-Fc fused ACE2 microbody. Importantly, this improvement in infectivity is observed with both wild-type spike protein as well as the D614G mutant. Our results hold important implications for the design and interpretation of similar LV pseudotyping-based studies.

Keywords: SARS-CoV-2, virus neutralization, spike lentiviral pseudotyping, nucleocapsid, ACE2-Fc

\section{INTRODUCTION}

The ongoing coronavirus disease 2019 (COVID-19) pandemic has provided a strong impetus for studies aimed at discovering and characterizing neutralizing antibodies or small molecule inhibitors targeted against the severe acute respiratory syndrome coronavirus 2 (SARS CoV-2). A major focus of these efforts has been the spike (S) glycoprotein of the virus, which mediates the viral entry into target cells by recognition and binding to the cell-surface receptor angiotensin-converting enzyme 2 (ACE2) (Huang et al., 2020; Sreepadmanabh et al., 2020).

Numerous studies have demonstrated that the spike protein may be employed to generate stably pseudotyped lentiviral, retroviral, and vesicular stomatitis virus particles (Crawford et al., 2020; Johnson et al., 2020; Plescia et al., 2020; Shang et al., 2020; Neerukonda et al., 2021). Given that live SARS CoV-2 is a biosafety level-3 agent, these pseudotyping approaches have greatly facilitated investigations undertaken within lower containment facilities. Importantly, this has also allowed for the screening and characterization of neutralizing antibodies against SARS CoV-2, as these primarily show reactivity against the spike protein (Tandon et al., 2020). Furthermore, such pseudoviruses are deployable as platforms for the large-scale screening of small molecule inhibitors 
and pharmacological agents which possess therapeutic potential against COVID-19 (Hu et al., 2020).

In this regard, capitalizing on the interaction of the $S$ protein with cellular ACE2, strategies have been reported which utilize chimeric ACE2 fused to the Fc region of human IgG as a potent neutralizing agent against spike-pseudotyped viruses (Lei et al., 2020). This arsenal now includes variations such as mutations to the catalytic region of ACE2 (thereby preventing undesirable side-effects during in vivo administration), and modified FC domain (Lei et al., 2020; Tada et al., 2020). However, a common feature of these studies is that they invariably adopt pseudoviruses which are enveloped by spike, but do not incorporate contributions from any other SARS CoV-2 proteins.

This point is particularly crucial in light of the manifold roles adopted by various proteins of the SARS $\mathrm{CoV}-2$, as has been highlighted in recent interactome studies (Gordon et al., 2020; Khorsand et al., 2020). Indeed, the specific effects of these genes continue to be investigated and represent a wide diversity of specialized functions. Building on this, we hypothesized that specific genes of the SARS CoV-2 could play a key role in enhancing the infectivity of viral particles. To probe this, we undertook an unbiased screen of twenty-four ORFs, NSPs, and structural protein genes of the SARS CoV-2 using spikepseudotyped lentiviral particles. Our observations implicated the $\mathrm{N}$ protein as an enhancer of viral infectivity for both the wild-type as well as the highly infectious D614G mutant of the spike glycoprotein. We further show that this enhancement of infectivity renders the viral particles less sensitive to ACE2Immunoglobulin chimera-mediated neutralization.

\section{METHODS}

\section{Cell Culture}

HEK293T (from ECACC) and ACE2-positive HEK293T were cultured in DMEM containing 10\% Fetal Bovine Serum (US origin certified serum), $1 \%$ penicillin-streptomycin, and $2 \mathrm{mM}$ L-glutamine (complete medium), all obtained from Gibco. The ACE2 expressing stable HEK293T cell line was generated by transduction with lentiviral particles followed by selection with hygromycin until the entire non-transduced population was eliminated. Expression of ACE2 was verified as described below.

\section{Plasmids}

The list of plasmids that were used in this study is provided in the Supplementary Information (refer to "Table S1: List of Plasmids") and are available upon request. pScalps-LuciferaseZsgreen was generated by cloning a firefly luciferase gene that was PCR amplified with primers incorporating the XhoI/EcoRI restriction sites. The resultant PCR product was digested and ligated in the pScalps Zsgreen plasmid using identical sites and the inserts were confirmed by Sanger sequencing.

ACE2 expressing lentiviral plasmid was generated by amplifying ACE2 encoding gene from the Addgene plasmid \#154987 and cloning into a modified pScalps lentiviral vector carrying the hygromycin selection marker. SARS CoV2 encoding genes (detailed below in "Table S1: List of Plasmids") were generously provided by the Nevan Krogan Lab in the lentiviral backbone pLV-TetONE, which were further subcloned into a non-viral pcDNA based custom-designed vector for expression of these gene in transient transfection assays. After cloning, each gene of interest preserved the Strep tag in-frame.

Site-directed mutagenesis was employed to generate a D614G mutant version of the SARS CoV-2 spike glycoprotein from the available sequence (Addgene, plasmid catalog \#155297). Using a pair of mutation-carrying primers (forward primer: 5' GGTGCAATTCACGCCCTGGTACAGCAC-3' and reverse primer: 5'-GTGCTGTACCAGGGCGTGAATTGCACC-3'), the plasmid was PCR amplified. The products were processed using a PCR cleanup kit (Macherey-Nagel), digested with Dpn1 (Thermofisher Scientific) overnight, and used to transform competent XL1-blue bacterial strain. Colonies obtained were screened, plasmids were sequenced (using the primer: 5'TCGGAAGGGACATCGCTGAT-3'), and a final clone matching the desired mutation was obtained. All the oligos for generation/sequencing of the plasmids used in this study were custom synthesized by Sigma-Aldrich.

\section{Virus Production and Quantification}

In general, lentiviral particles were produced by calcium phosphate transfection of HEK293T with $8 \mu \mathrm{g}$ of transfer vector, $6 \mu \mathrm{g}$ of packaging plasmid (psPAX2) and $2 \mu \mathrm{g}$ of envelope plasmid (spike-expressing plasmid or pMD2.g). The culture medium was replaced at $16 \mathrm{~h}$-post transfection. Lentiviral vector-containing supernatant was collected $48 \mathrm{~h}$ after transfection and was centrifuged and filtered through $0.22 \mu \mathrm{m}$ syringe filters. The infectivity assay was performed after normalizing reverse transcriptase (RT) units obtained from an SGPERT assay as described earlier (Pizzato et al., 2009). Briefly, the target cells were infected in quadruplicates (or at least triplicate) with up to 125 -fold dilutions and the infectivity was acquired from the dilutions in the linear range as reported previously (Chande et al., 2016). Expression of GFP or Luciferase as a quantitative measure of infection was acquired using SpectraMax-i3X plate reader (Molecular Devices, USA) or High-content imaging platform (CellInsight CX7, ThermoFisher Scientific).

The SARS CoV-2 genes' screen was performed by producing spike pseudotyped lentiviruses from HEK293T cells that were seeded in 12-well plate. Next day cells were transfected with pScalps luciferase Zsgreen (800 ng), psPax2 (600 ng), spike expression plasmid (200 ng), and either vector control or ORF carrying vector (300 ng) by the calcium phosphate method. Culture medium was replaced with fresh medium $16 \mathrm{~h}$ after transfection. Spike pseudotyped viruses were collected after $48 \mathrm{~h}$ of transfection. Supernatant collected was centrifuged at $1200 \mathrm{xg}$ for $5 \mathrm{~min}$ and filtered using a $0.22 \mu \mathrm{m}$ syringe filter.

Spike pseudotyped viruses were then used to transduce ACE2 expressing HEK293T cells to check the infectivity level by either GFP positive cell count and or Luciferase assay.

To quantify the RT units, $5 \mu$ l viruses were lysed in a $5 \mu$ lysis buffer and diluted with a core buffer to make volume $100 \mu \mathrm{l}$. 
A $10 \mu \mathrm{l}$ diluted viral lysate was mixed with an equal volume of $2 \mathrm{X}$ reaction buffer and the SGPERT assay was performed.

For immunoblotting of proteins incorporated into lentiviral particles, the lentiviral particles-containing supernatant was concentrated on sucrose cushion by ultracentrifugation at 100,000Xg for 2 hours at $4^{\circ} \mathrm{C}$ (Chande et al., 2016). Viral pellet obtained was resuspended in the Laemmli buffer containing $50 \mathrm{mM}$ TCEP as a reducing agent.

Murine Leukemia Virus (MLV)-derived vectors were produced, from HEK293T cells seeded in a 12-well plate format, by transfecting $800 \mathrm{ng}$ of MLV-NCA Zsgreen plasmid with 200 ng of VSV-G envelope or spike glycoprotein encoding plasmid (200ng), either in the presence or absence of $\mathrm{N}$-encoding plasmid (200ng). The reverse transcriptase units were quantified by SGPERT assay and ACE2-positive HEK293T served as target cells. The infection was quantified by counting the GFP positive cells using the SpectraMax-i3X plate reader.

\section{Gene Expression}

To check the expression of ACE2 in ACE2-transduced HEK293T cells we isolated the RNA from HEK293T and ACE2-transduced HEK293T by Trizol method. The cDNA was synthesized from $1 \mu \mathrm{g}$ of RNA using oligodT primers obtained from both the cells. To quantify the level of ACE2 expression, RT-PCR was performed using ACE2 specific primers 5'-GGGATCAGA GATCGGAAGAA-3' forward and 5'-AGGAGGTCTGAA CATCATC-3' reverse and GAPDH as control with primers 5'TGGAGAAGGCTGGGGCTCATTTGCA-3' forward and 5'CATACCAGGAAATGAGCTTGACAA-3’.

Quantitative real-time (qRT-PCR) for determining the expression of spike glycoprotein both in the absence and presence of $\mathrm{N}$ was carried out by isolating the DNA-free RNA from transfected cells as described above, and then processed for qRT-PCR using the spike-specific primers 5'- GCAACG TGACCTGGTTCCAC-3' in forward and 5'- CGATCAGC AGGCTCTGGGTC-3' in reverse.

\section{Luciferase Assay and GFP Cell Count}

Luciferase assay was performed in 96-well plates to quantify level of transduction by spike pseudotyped viral particles. Equal numbers of ACE2+ HEK293T cells were seeded in 96-well plates (10,000 cells/well) $24 \mathrm{~h}$ before transduction and incubated with the various pseudotyped viruses for $48 \mathrm{~h}$. Zsgreen (GFP) cell count was first scored using the SpectraMax-i3X system (Molecular Devices). Media was aspirated from each well postGFP acquisition and cells were lysed by treatment with $100 \mu \mathrm{l}$ lysis buffer for 20 minutes at room temperature. Luciferase readings were measured using SpectraMax-i3X by injecting 50 $\mu \mathrm{l}$ of substrate buffer in $50 \mu \mathrm{l}$ of cell lysate in 96-well white plates. Specifics of buffer composition may be found below in the section titled "Buffers and common reaction mixtures".

\section{Western Blotting}

For Western blot-based analysis, samples were prepared in a $4 \mathrm{X}$ Laemmli buffer and run on either $8 \%, 12.5 \%$, or $15 \%$ tricine gels for electrophoresis depending upon the molecular weight range being detected by this method. Following this, gels were electro blotted on the PVDF membrane (Immobilon-FL, MerckMillipore). Blocking of membranes was carried out by incubation with either a $5 \%$ BSA solution or the proprietary Odyssey Blocking Buffer (LI-COR Biosciences) for one hour, followed by both primary and secondary antibody incubations for one hour each at room temperature, each of which were followed by three washes of five minutes each.

Detection of p24, beta-actin, SARS CoV-2 spike glycoprotein, Strep-tagged SARS CoV-2 genes, and ACE2-IgFc was carried out using mouse anti-p24 (NIH ARP), rabbit anti-beta actin (LICOR Biosciences, Cat\# 926-42210, RRID: AB_1850027), mouse anti-spike (Cat\# ZMS1076, Sigma Aldrich), mouse anti-Strep (Qiagen, Cat\# 34850), and mouse anti-Histidine (Invitrogen, Cat\# MA1-21315), respectively, as primary antibodies. Secondary antibodies used were either IR dye 680 goat antimouse, IR dye 800 goat mouse, or IR dye 800 goat anti-rabbit (LI-COR Biosciences Cat\# 925-68070, RRID: AB_2651128, and LI-COR Biosciences Cat\# 925-32211, RRID: AB_2651127).

\section{Flow Cytometry}

HEK293T cells were transfected with either pScalps Luciferase Zsgreen alone or along with $\mathrm{N}$ protein. Cells were harvested after 48 hours, fixed in $4 \%$ paraformaldehyde, and prepared for flow cytometry after removing the fixative. As a control, HEK293T cells were also processed in a similar manner.

\section{ACE2-IgFc Microbody Constructs}

Genomic DNA isolated from HEK293T cells using the Macherey-Nagel Nucleospin kit (\#740952.50) was used as a template for PCR amplifying the Fc region of human IgG. The forward primer 5'-CAGCACCTGAACTCCTGGGGGGACCG3' and reverse primer 5'-CCTTTGGCTTTGGAGAT GGTTTTC-3' was used to amplify the first exon encoding the Fc region. The forward primer 5'-AGGGCAGCCCCGAG AACCACAGGTG-3' and reverse primer 5'-TTTACCCGGAG ACAGGGAGAGGCT-3' was used to amplify the second exon encoding the $\mathrm{Fc}$ region. Both fragments were individually amplified further using the combination of forward and reverse primers as 5'-GAAAACCATCTCCAAAGCCAAAGGGCAGCC CCGAGAACCACAGGTG-'3' and 5'-TATATATTCTAGAT TAATGGTGATGGTGATGATGGCCGCCTTTACCCGG AGACAGGGA-3' for the first exon's amplicon and 5'ATATATCTCGAGGACAAAACTCACACATGCCCACCGT GCCCAGCACCTGAACTCCTG-3' and 5'-CCTTTGGCT TTGGAGATGGTTTTC-3' for the second exon's amplicon, respectively. Finally, both these fragments were combined using the forward primer 5'-ATATATCTCGAGGAC AAAACTCACACATGCCCACCGTGCCCAGCACCTG AACTCCTG-3' and reverse primer 5'-TATATATTCTAGA TTAATGGTGATGGTGATGATGGCCGCCTTTACCCGG AGACAGGGA-3' to generate the final Fc fragment, which was cloned into pTZ57R.

Addgene (\#154987) was used as template for ACE2 amplification, using the forward primer 5'-GAACAAGAATT CTTTTGTGGGA-3' and reverse primer 5'-TTTGTCCT CGAGGGAAACAGGGGGCTGGTTAG-3' a 421 bp fragment of the same was amplified and cloned into pTZ57R. 
Using an EcoRI/XhoI digestion, the ACE2 fragment was combined upstream of the Fc, and the combined fragment was ligated into the ACE2 containing plasmid using a EcoRI/XbaI digestion to yield the final ACE2-IgFc construct. The construct was verified with Sanger sequencing (refer to "Table S2: Sequences of ACE2-IgFc").

\section{Biochemical Characterization of ACE2-IgFc Microbody}

HEK293T cells were transfected with the plasmid encoding ACE2-IgFc ( $5 \mu$ g plasmid in a $35 \mathrm{~mm}$ dish). As a control, $5 \mu \mathrm{g}$ of pcDNA3.1BS(-) was also transfected. Media was changed twelve hours post-transfection and fresh DMEM (without FBS) was added to the dishes. Culture media was collected both 48and 72-hours post-transfection, and samples were prepared in either $8 \%$ or $2 \%$ SDS-containing 4 X Laemmli buffer (with and without TCEP added, respectively) for SDS-PAGE. $8 \%$ tricine gels were run, and analyzed using Western Blotting. The amount of ACE2 present in the supernatant was determined by comparison with standard concentrations of pure BSA as ascertained by band density analysis following silver staining of SDS-PAGE gels. For Western Blotting, the proteins were transferred to a PVDF membrane, blocked in a 5\% BSA solution in TBS, and primary mouse-derived anti-Histidine antibodies were incubated with the blot in a 1:4000 dilution, followed by goat-derived anti-mouse antibodies in a 1:5000 dilution.

\section{Pull-Down Assay Using ACE2-IgFc}

Thirty microliters of protein-G Dynabeads (Thermofisher Scientific Cat \#10003D) were first equilibrated by washing with $10 \%$ FBS-containing DMEM. Following this, these were incubated with gentle mixing by inversion for 15 minutes with $100 \mu \mathrm{l}$ of ACE2-IgFc containing supernatant (equivalent of 10 $\mu \mathrm{g} / \mathrm{ml}$ ). This was split into three equal fractions of $10 \mu \mathrm{l} \mathrm{each}$. The beads-containing mixtures then were immobilized on a magnetic rack, supernatant was aspirated off, and replaced with $100 \mu \mathrm{l}$ (10mU equivalent/mL RT) of culture supernatant carrying either bald viruses or viruses pseudotyped with VSV-G or Spike glycoproteins. Mixing was carried out for a period of 15 minutes, following which these were placed back on the magnetic rack, supernatant was aspirated, and the beads washed twice with SGPERT Core Buffer - first with $500 \mu \mathrm{l}$ and then with $100 \mu$ l. Finally, $20 \mu \mathrm{l}$ of RT Lysis Buffer was added and the reaction was incubated at room temperature. Following this, the reaction was 10 -fold diluted using core buffer. The resulting supernatant was collected after immobilizing the beads and processed for an SGPERT Assay.

\section{Neutralization Assay}

To quantify the amount of ACE2-IgFc required to inhibit the infection in ACE2+ HEK293T cells, we treated $100 \mu \mathrm{l}$ of spike pseudotyped virus (normalized to RT units) with a variable amount of ACE2-IgFc diluted in the growth medium. The mixture was incubated for fifteen minutes at room temperature, following which each sample was used to transduce ACE2+ HEK293T cells seeded in 96-well plates.
Luciferase assay was performed 48 hours post-transduction, with readings acquired using the SpectraMax-i3X plate reader.

\section{Buffers and Common Reaction Mixtures}

TBS (Tris-Buffered Saline) was prepared by mixing a $500 \mathrm{mM}$ solution of Tris ( $\mathrm{pH} 7.4$ ) with water and $\mathrm{NaCl}$ (up to a final concentration of $1500 \mathrm{mM}$ ). For making TBS-T, Tween-20 (Sigma Aldrich) was added to TBS up to a final concentration of $0.01 \%$.

For making $4 \mathrm{X}$ Laemmli buffer, components were mixed to achieve the final concentrations as follows: $200 \mathrm{mM}$ Tris $(\mathrm{pH}$ 6.8), 40\% glycerol (Sigma Aldrich), $50 \mathrm{mM}$ Tris(2-carboxyethyl) phosphine (TCEP) (Thermo Scientific), 8\% SDS (Sigma Aldrich), and water up to $50 \mathrm{ml}$. Non-reducing buffer conditions were achieved by removing TCEP from the above formula and decreasing the amount of SDS added to a final concentration of $2 \%$.

A proprietary Odyssey Buffer for blocking of membranes and dilution of antibody stocks was used as supplied (LICOR Biosciences).

Luciferase assay buffers were prepared as a lysis buffer and substrate buffer. Lysis buffer required a final concentration of components as $1 \%$ Triton X-100 (Sigma Aldrich), $25 \mathrm{mM}$ Tricine (VWR), $15 \mathrm{mM}$ of potassium phosphate (at $\mathrm{pH} 7.8$ ), $15 \mathrm{mM} \mathrm{MgSO}, 4 \mathrm{mM}$ EGTA, and $1 \mathrm{mM}$ DTT, with water to make up the solution. Substrate buffer required further addition of $1 \mathrm{mM}$ DTT (VWR Scientific), $1 \mathrm{mM}$ ATP (Sigma Aldrich), and $0.2 \mathrm{mM}$ D-Luciferin (Cayman) to the aforementioned lysis buffer. All fine chemicals were procured from Sigma-Aldrich unless otherwise indicated.

Harvesting of cell samples for Western blotting was carried out using the RIPA lysis buffer (Lysis Buffer, 2017).

\section{Software and Statistical Analysis}

All graphs were generated using GraphPad Prism (version 9.0). Statistical analysis was carried out using the in-built algorithms bundled with the software. Two-tailed unpaired t-test was used to calculate the statistical significance shown for all pairwise comparisons, where ${ }^{\star * *}=\mathrm{p}<0.0001,{ }^{* *}=\mathrm{p}<0.001$, $^{\star *}=$ $\mathrm{p}<0.01$, and ${ }^{\star}=\mathrm{p}<0.05$. Specific portions of images were produced using Biorender. Band intensities of Western blots were calculated using the in-built analytical tools from the Image Studio Lite Ver. 5.2 (LI-COR Biosciences).

\section{RESULTS}

A custom-designed transfer vector (Figure 1A), termed pScalps Luciferase-Zsgreen, as well an ACE2-expressing HEK293T cell line (Figure 1B) were generated for this study. To investigate the effect of various SARS CoV-2 genes on viral infectivity, we undertook an unbiased screen, as outlined by the schematic in Figure 1C. Briefly, HEK293T producer cells were transfected with plasmids encoding the codon-optimized $\mathrm{S}$ protein (with a nineteen amino-acid deletion at the $\mathrm{C}$ terminal), the packaging plasmid psPAX2, and the pScalps Luciferase-Zsgreen reporter plasmid. Alongside this, each one of the twenty-four SARS CoV2 genes was separately co-transfected with the above. 
A

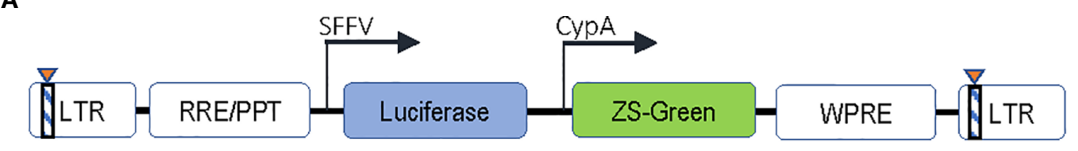

C

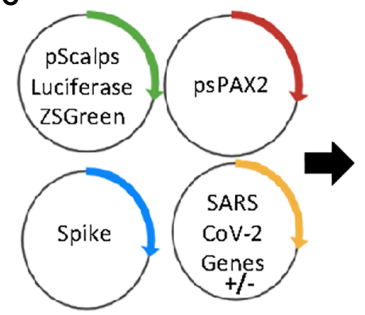

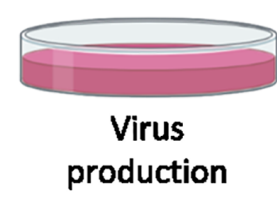

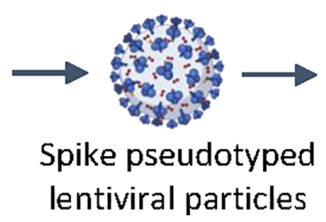

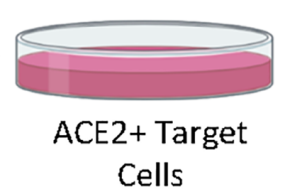

B
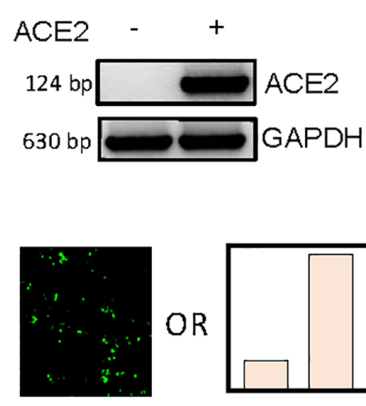

GFP

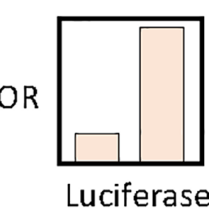

FIGURE 1 | Experimental setup and constructs. (A) Schematic representation of the pScalps Luciferase Zsgreen lentiviral reporter construct. (B) ACE2 expression from the hygromycin selected HEK293T cell line transduced with a ACE2 gene carrying lentiviral vector. (C) Schematic depiction of the SARS CoV-2 genes' screen. Transfection with an empty vector served as control. Readout from the screen was obtained in the form of firefly luciferase activity or GFP-positive cell count.

ACE2-expressing HEK293T cells were transduced with viruses produced in the presence of indicated SARS CoV-2 genes, and the extent of infectivity was gauged as a measure of both GFP-positive cell count and firefly luciferase activity 48 hours post-infection (Figure 2A). Expression of all the SARS $\mathrm{CoV}-2$ genes was verified (Supplementary Figure 1A) and the effect of this expression was also checked on the lentiviral capsid levels by western blotting from the producer cell lysates (Figure 2B). While non-uniform expression pattern of a minor subset of these genes could be a limitation, we identified the nucleocapsid $(\mathrm{N})$ protein as a prominent enhancer of spike-pseudovirus infectivity. We scored the effects on infectivity in conditions where the co-expression of SARS CoV-2 genes increased the infectivity without an apparent effect on the lentiviral capsid levels (Figure 2B). The particle infectivity enhancement by $\mathrm{N}$ was also discernible with a GFP reporter in the ACE2-postive target cells suggesting this assay reliably measures transduction events (Figure 2C). Additional control experiments were performed to exclude the influence of $\mathrm{N}$ protein on the reporters (Figures 2D, E) which helped determine that there is no significant effect of the $\mathrm{N}$ protein on either of these. Additionally, the infectivity-enhancement effect of $\mathrm{N}$ was not observed in the case of VSV-G pseudotyped lentiviral particles (Supplementary Figure 1B). We also employed an MLV vector to determine whether the observed phenotype was specific to HIV-1 core-based particles. While VSV-G pseudotyped MLV virions did not show any $\mathrm{N}$-dependent infectivity enhancement, spike-MLV pseudovirions did mirror the enhancement in infectivity observed with the lentiviral particles (Figure $2 \mathbf{F}$ and Supplementary Figure 1C). Further, based on recent reports of a relatively more infectious D614G spike glycoprotein variant (Zhang et al., 2020), we decided to screen the same to assess the effect of $\mathrm{N}$ protein. Cellular expression of the mutant protein at levels comparable with the wild-type spike was confirmed by Western blotting (Supplementary Figure 1D). Consistent with previous reports, the mutant strain produced pseudoviruses which were more infectious as compared to wild-type spikepseudovirions (Figure 2G and Supplementary Figure 1E). On top of this, inclusion of the $\mathrm{N}$ protein during particle production allowed for an even higher extent of infectivity, which reaffirmed its role in a manner consistent with the above results. Cumulatively, these results appear to implicate the viral $\mathrm{N}$ protein as an intrinsic enhancer of lentiviral spike-pseudotyped particle infectivity.

Next, we asked if such an enhancement of particle infectivity would impact the ability of neutralizing agents to block these particles from infecting host cells. Capitalizing on the interaction of the $S$ protein with cellular ACE2, we generated a SARS CoV-2 entry receptor-based synthetic microbody consisting of a soluble ACE2 domain fused with the Fc region of human IgG (Figure 3A), which also carried a C-terminal 6XHistidine tag. Extracellular expression, as well as the ability of ACE2-IgFc to dimerize under the experimental conditions that retained disulphide linkage, were detected by Western blotting (Figure 3B). These experiments also confirmed the presence of an intact Histidine tag. Furthermore, we tested the specific interaction of the ACE2-IgFc with spike-pseudotyped LV particles by a pulldown experiment wherein the on-bead capture of viral particles using protein G-bound ACE2-IgFc resulted in a $\sim 10,000$-fold enrichment of spike pseudotyped viruses over VSV-G pseudotyped or non-enveloped (bald) lentiviral particles (Figure 3C). This also suggests that Fc configuration remained intact after fusion with ACE2 thereby facilitating the immobilization of the microbody on the protein $G$ magnetic beads for virion capture. In sum, these results established that the ACE2-IgFc molecule is stably expressed and demonstrates a high affinity specifically towards the spike-laden particles.

Following this, we wished to ascertain the relative titre of ACE2-IgFc required to effectively restrict the infection of ACE2+ target cells, under the influence of the $\mathrm{N}$ protein. Briefly, lentiviral particles pseudotyped with spike (with and without co-transfection with $\mathrm{N}$ ) or bald particles lacking envelope were 


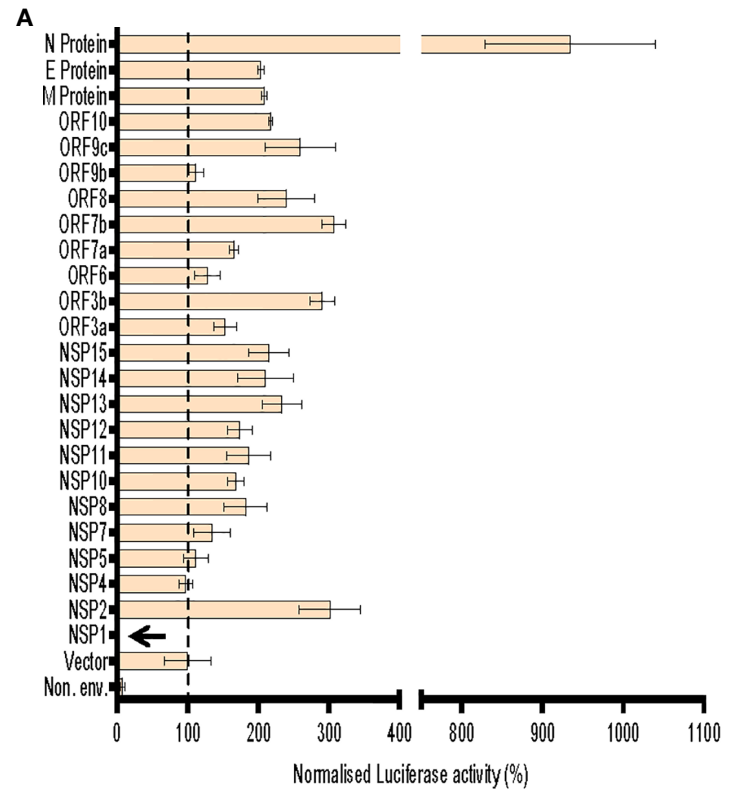

B

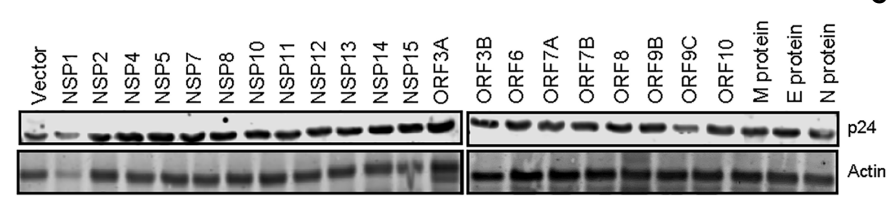

D

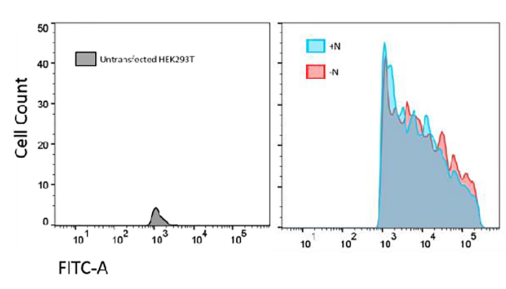

$\mathbf{F}$

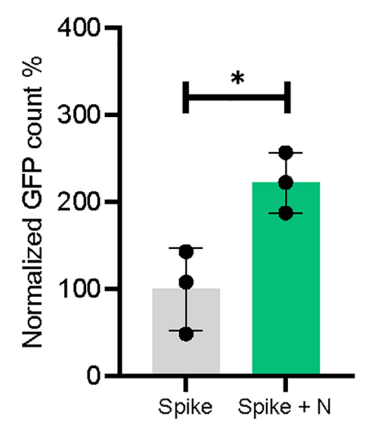

E

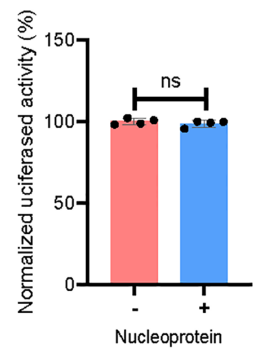

G

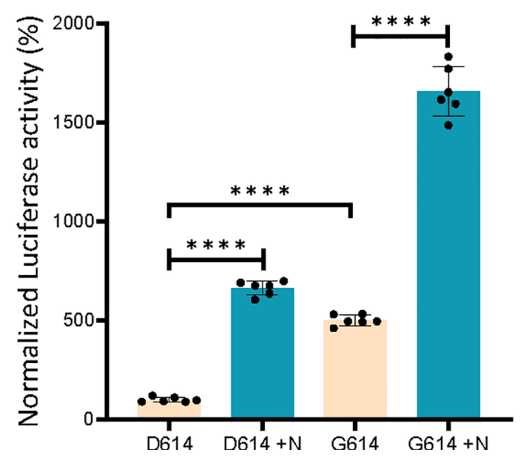

FIGURE 2 | SARS CoV-2's N protein enhances retroviral spike-pseudotyped particle infectivity. (A). Results from the screen of SARS CoV-2 genes, either by quantifying luciferase activity (left panel) or GFP positive cell count (right panel). Vector normalized values for both readout methods has been shown, such as to indicate the percentage change relative to the baseline (indicated by the dotted line). Background signal levels are indicated by the non-enveloped (Non. env.) condition. $\mathrm{n}=4 \pm$ SD. (B) p24 levels from the virus-producing cells for all the SARS CoV-2 genes were detected by Western blotting. Actin served as loading control. (C) Microscopy images for the infected cells with indicated conditions (scale $100 \mu \mathrm{m}$ ). (D) Representative flow cytometric analysis of the effect of $\mathrm{N}$ protein on Zsgreen expression, using populations transfected with either the reporter plasmid alone (red fraction) or along with the N protein (cyan fraction). (E) Quantification of the luciferase gene expression from the pScalps Luciferase Zsgreen reporter plasmid with and without the presence of $N$ protein. $n=4 \pm S D$. (F) Infectivity of MLV vector pseudotyped with the spike glycoprotein for both $\mathrm{N}$-exclusive and $\mathrm{N}$-inclusive conditions, normalized to the former. $\mathrm{n}=3 \pm \mathrm{SD}$ (G) Comparison between the infectivity of both wild-type spike and the D614G mutant, with and without N protein co-transfection, normalized to the wild-type spike-only condition $n=6 \pm S D$. ${ }^{*} p<0.05$; ${ }^{\star \star \star *} p<0.0001$; NS, Non significant. 


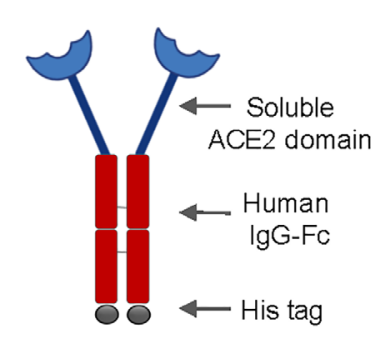

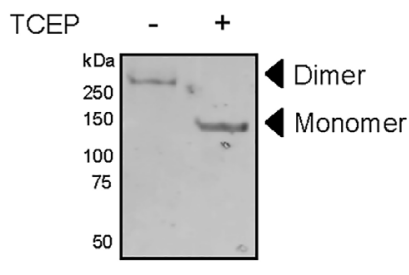

C

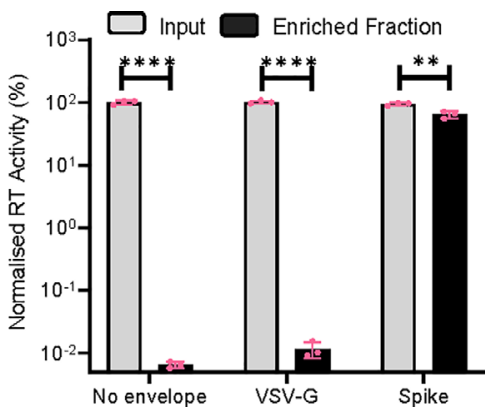

FIGURE 3 | Characterization of the ACE2-IgFc microbody. (A) Schematic of the ACE2-IgFc neutralizing microbody. (B) Effect of a reducing agent (TCEP) on the migration of ACE2-IgFc on the gel. Anti-His antibody indicating monomeric vs dimeric states of the protein visualized by western blotting from the cell culture supernatant. (C) Protein G-bound ACE2-IgFC and the enrichment of virus particles. The RT activity of the input VSV-G or Spike particles was normalized to the non-enveloped particles, set to $100 \% . n=3 \pm S D .{ }^{\star \star} p<0.01 ;{ }^{* \star \star \star} \mathrm{p}<0.0001$.

generated, normalized to the RT units, incubated with ACE2IgFc according to the indicated concentrations, and used to transduce ACE2+ HEK293T target cells. Luciferase activity assay, as a quantitative and sensitive indicator of transduction events, revealed the ability of ACE2-IgFc to impair the infectivity of spike pseudoviruses in a dose-dependent manner. Furthermore, results obtained herein demonstrate the requirement for a significantly higher neutralizing titre of ACE2-IgFc microbodies in the case of $\mathrm{N}$-enhanced particles as opposed to those generated solely by the spike glycoprotein in order to elicit similar levels of inhibition (Figure 4A). Additional experiments using D614G mutant also indicated that the $\mathrm{N}$ inclusive particles would require a higher amount of the neutralizing agent in comparison to $\mathrm{N}$-exclusive counterpart (Supplementary Figure 1F). The higher requirement of neutralizing agent was not a result of higher particle counts as the inoculum was normalised to the reverse transcriptase units.

To better comprehend these observations, we envisaged a modification of virus particles by $\mathrm{N}$ that plausibly impacted the virion quality, rather than the quantity. To better understand this, we asked if $\mathrm{N}$ protein directly impacted the spike protein itself in order to effect infectivity enhancement. Accordingly, we first analyzed the effect of $\mathrm{N}$ on spike at the mRNA level using both quantitative RT-PCR and semiquantitative gel-based methods and found no significant upregulation of the same (Figure 4B). Next, the viral particles produced under indicated conditions were pelleted on a sucrose cushion as described earlier (Chande et al., 2016). Biochemical analysis of the virus pellet and the corresponding cell lysates by Western blotting revealed that $\mathrm{N}$ improved the steady-state levels of spike in the producer cell lysates. The enhancement of spike signal was noticeably more prominent from the virions (Figures $4 \mathrm{C}$, D). The augmentary effects of $\mathrm{N}$ appear to be specific in nature, given that exclusively lentiviral components do not show any apparent changes in expression. Altogether, these experiments indicated that $\mathrm{N}$ protein likely promoted the incorporation and retainment of spike protein in the virions, thereby improving the particle quality and its infectivity.

\section{DISCUSSION}

Considering the technical difficulties associated with studying live, replication-competent native SARS CoV-2, the adoption of lentiviral or retroviral pseudotyping-based systems has enabled a more widespread and accessible investigation of this pathogen. Significant advances have been achieved in recent times, ranging from optimization of pseudotyping protocols to the application of these for screening entry inhibitors (Hu et al., 2020). The roles of structural proteins such as $\mathrm{M}, \mathrm{E}$, and $\mathrm{N}$ in assisting viral assembly and particle formation have also been explored (Plescia et al., 2020). Along similar lines, leading studies have deciphered the manifold cellular associations and interactions of various SARS CoV-2 genes, in processes as diverse as host translation inhibition, disruption of splicing, immune evasion, and protein trafficking, among others (Banerjee et al., 2020; Schubert et al., 2020; Xia et al., 2020; Flower et al., 2021).

In the present study, we have evaluated the potential of twenty-four SARS CoV-2 genes to elicit superior infectivity levels as compared to what may be achieved by mere pseudotyping with the spike glycoprotein. Chief among these has been the nucleocapsid $(\mathrm{N})$ protein. While a previous study has established the crucial role played by $\mathrm{N}$ in viral genome processing and nucleocapsid assembly (Carlson et al., 2020), to the best of our knowledge there have been no prior reports specifically highlighting its ability to elevate lentiviral spike pseudovirus infectivity. It bears mentioning that similar to existent reports (Min et al., 2020; Schubert et al., 2020; Thoms et al., 2020; Lapointe et al., 2021; Zhang et al., 2021), the Nsp1 protein presumably exerts a detrimental effect on the host cell's protein translation, as indicated by the almost imperceptible extent of infectivity observed in its case (Figure 2A).

Our assessment of pseudoviruses generated from both $\mathrm{N}$ exclusive and $\mathrm{N}$-inclusive backgrounds directly implicates $\mathrm{N}$ as a potent enhancer of virion quality and infectivity, an effect which extends over both the wild-type spike protein as well as the D614G mutant. Similar to the proposed infectivity enhancing mechanism for the D614G variant, which relies on reduced S1 
A

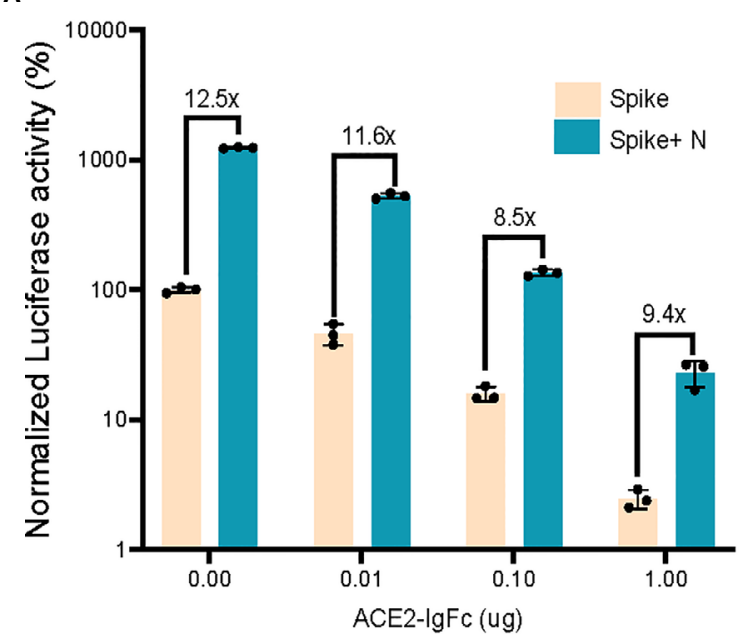

B

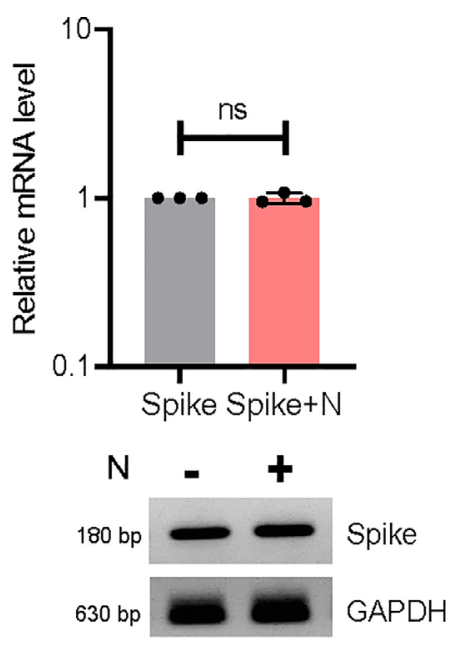

C

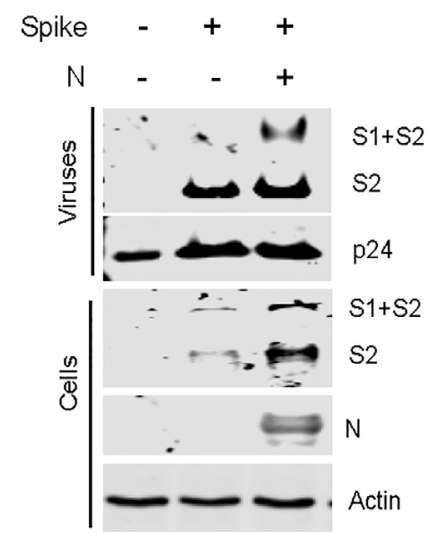

D
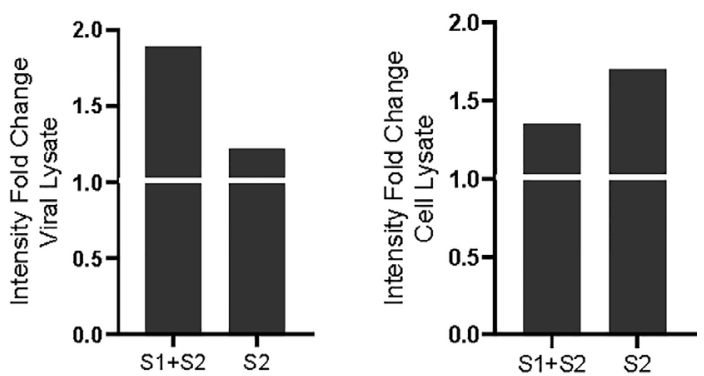

FIGURE 4 | Neutralizing effects of ACE2-IgFC on the N-enhanced spike lentiviral particles and biochemical characterization of the virions (A) Neutralization assay using ACE2-IgFc pre-treated lentiviral particles with indicated amount of ACE2-IgFC (on the $x$-axis) for the lentiviral particles produced in the presence of indicated genes. The particles were normalized to the reverse transcriptase units obtained from the SGPERT assay. Luciferase activity obtained from non-enveloped particle transduction was considered as baseline, and the data represented has been normalized to the spike-only condition without the addition of ACE2-IgFc. The lines represent extent of residual infectivity (expressed as $x$-fold), post-treatment of ACE2-lgFc, with respect to $N$-exclusive particles $n=3 \pm S D$. (B) RT-PCR analysis of the $m R N A$ fraction from cells transfected with either spike or spike $+N$ protein. Both quantitative RT-PCR and representative semi-quantitative PCR results are shown. $n=3 \pm$ SD. (C) Biochemical analysis of the virus particles and the corresponding cell lysates. The viral proteins were visualized by western blotting using antibodies raised against S2 domain of SARS CoV2 spike. (D) Densitometric analysis of the band intensities were obtained by normalizing the S1+S2 and S2 bands to either the respective p24 levels for viral lysate or the Actin levels for cell lysate and calculating the fold change of the normalized intensities of bands in the $\mathrm{N}$-inclusive condition to the spike-only condition. The N protein and p24 were detected using Anti-Strep tag and anti-p24 antibody respectively. Actin served as loading control. $\mathrm{n}=3$, representative blots are shown. NS, Non significant.

shedding and increased spike protein retainment in virions (Zhang et al., 2020), the $\mathrm{N}$-dependent enrichment of spike glycoprotein within viral fractions we demonstrated lends support to this hypothesis. The identification of $\mathrm{N}$ in this particular role may be a telling example of how the SARS CoV-2 adopts a host of varied and as-yet incompletely explored strategies to drive its pathogenesis. Future extensions of this work may gain clinically relevant insights by including sera from vaccinated individuals or convalescent plasma from recovered patients to assess the neutralizing potential of these against similarly modified spike pseudoviral systems.
In sum, our results underscore the importance of factoring in additional contributions from the other viral genes while undertaking lentiviral vector pseudotyping with the spike glycoprotein of the SARS CoV-2. While existing studies have managed to develop and validate platforms for the screening of neutralizing antibodies and inhibitory chemical compounds, we believe that our results highlight the necessity of incorporating additional genetic elements which have been shown to boost viral infectivity. Furthermore, putative therapeutic candidates currently being scrutinized in various clinical studies would benefit from lentiviral pseudotyping assays that incorporate 
additional virion components during the large-scale screening stage.

\section{DATA AVAILABILITY STATEMENT}

The original contributions presented in the study are included in the article/Supplementary Material. Further inquiries can be directed to the corresponding author.

\section{AUTHOR CONTRIBUTIONS}

Performed research and analyzed data: TM, SM, PR, AS, and AC. Contributed reagents: AK. Wrote the paper: AC and SM. Conceived, designed research, and supervision: AC. Contributed equally: TM and SM. All authors contributed to the article and approved the submitted version.

\section{REFERENCES}

Banerjee, A. K., Blanco, M. R., Bruce, E. A., Honson, D. D., Chen, L. M., Chow, A., et al. (2020). SARS-Cov-2 Disrupts Splicing, Translation, and Protein Trafficking to Suppress Host Defenses. Cell 183, 1325-1339.e21. doi: 10.1016/j.cell.2020.10.004

Carlson, C. R., Asfaha, J. B., Ghent, C. M., Howard, C. J., Hartooni, N., Safari, M., et al. (2020). Phosphoregulation of Phase Separation by the SARS-Cov-2 N Protein Suggests a Biophysical Basis for its Dual Functions. Mol. Cell 80, 10921103.e4. doi: 10.1016/j.molcel.2020.11.025

Chande, A., Cuccurullo, E. C., Rosa, A., Ziglio, S., Carpenter, S., and Pizzato, M. (2016). S2 From Equine Infectious Anemia Virus is an Infectivity Factor Which Counteracts the Retroviral Inhibitors SERINC5 and SERINC3. Proc. Natl. Acad. Sci. 113, 13197-13202. doi: 10.1073/pnas.1612044113

Crawford, K. H. D., Eguia, R, Dingens, A. S., Loes, A. N., Malone, K. D., Wolf, C. R., et al. (2020). Protocol and Reagents for Pseudotyping Lentiviral Particles With SARS-CoV-2 Spike Protein for Neutralization Assays. Viruses 12 (5), 513. doi: $10.3390 / v 12050513$

Flower, T. G., Buffalo, C. Z., Hooy, R. M., Allaire, M., Ren, X., and Hurley, J. H. (2021). Structure of SARS-CoV-2 ORF8, a Rapidly Evolving Immune Evasion Protein. Proc. Natl. Acad. Sci. U. S. A. 118 (2), e2021785118. doi: 10.1073/ pnas. 2021785118

Gordon, D. E., Jang, G. M., Bouhaddou, M., Xu, J., Obernier, K., White, K. M., et al (2020). A SARS-CoV-2 Protein Interaction Map Reveals Targets for Drug Repurposing. Nature 583, 459-468. doi: 10.1038/s41586-020-2286-9

Huang, Y., Yang, C., Xu, X., Xu, W., and Liu, S. (2020). Structural and Functional Properties of SARS-CoV-2 Spike Protein: Potential Antivirus Drug Development for COVID-19. Acta Pharmacol. Sin. 41, 1141-1149. doi: 10.1038/s41401-020-0485-4

Hu, J., Gao, Q., He, C., Huang, A., Tang, N., and Wang, K. (2020). Development of Cell-Based Pseudovirus Entry Assay to Identify Potential Viral Entry Inhibitors and Neutralizing Antibodies Against SARS-Cov-2. Genes Dis. 7, 551-557. doi: 10.1016/j.gendis.2020.07.006

Johnson, M. C., Lyddon, T. D, Suarez, R., Salcedo, B., LePique, M., Graham, M., et al. (2020). Optimized Pseudotyping Conditions for the SARS-COV-2 Spike Glycoprotein. J. Virol. 94 (21), e01062-20. doi: 10.1128/JVI.01062-20

Khorsand, B., Savadi, A., and Naghibzadeh, M. (2020). Sars-CoV-2-human Protein-Protein Interaction Network. Inform. Med. Unlocked 20, 100413. doi: 10.1016/j.imu.2020.100413

Lapointe, C. P., Grosely, R., Johnson, A. G., Wang, J., Fernández, I. S., and Puglisi, J. D. (2021). Dynamic Competition Between SARS-CoV-2 NSP1 and mRNA on the Human Ribosome Inhibits Translation Initiation. Proc. Natl. Acad. Sci. U. S. A. 118 (6), e2017715118 doi: 10.1073/pnas.2017715118.

\section{ACKNOWLEDGMENTS}

This work was supported by the intramural funds, a Department of Biotechnology (DBT) grant (BT/PR26013/GET/119/191/ 2017), and the Wellcome Trust/DBT India Alliance Fellowship [grant number IA/I/18/2/504006 awarded to AC]. TM and PR are supported by a fellowship from the MHRD. AC is a recipient of the Innovative Young Biotechnologist Award from the DBT. Authors are grateful to Nevan Krogan, Massimo Pizzato, Jeremy Luban, Sonja Best, Raffaele De Francesco, Didier Trono, Sunando Datta, and the NIH AIDS Reagent Program for the reagents and cell line.

\section{SUPPLEMENTARY MATERIAL}

The Supplementary Material for this article can be found online at: https://www.frontiersin.org/articles/10.3389/fcimb.2021. 663688/full\#supplementary-material

Lei, C., Qian, K., Li, T., Zhang, S., Fu, W., Ding, M., et al. (2020). Neutralization of SARS-CoV-2 Spike Pseudotyped Virus by Recombinant ACE2-Ig. Nat. Commun. 11, 2070. doi: 10.1038/s41467-020-16048-4

Lysis Buffer, R. I. P. A. (2017). RIPA Lysis Buffer. Cold Spring Harb. Protoc. 2017, pdb.rec101428 doi: 10.1101/pdb.rec101428.

Min, Y.-Q., Mo, Q., Wang, J., Deng, F., Wang, H., and Ning, Y. J. (2020). SarsCoV-2 Nsp1: Bioinformatics, Potential Structural and Functional Features, and Implications for Drug/Vaccine Designs. Front. Microbiol. 11, 587317. doi: $10.3389 /$ fmicb.2020.587317

Neerukonda, S. N., Vassell, R., Herrup, R., Liu, S., Wang, T., Takeda, K., et al. (2021). Establishment of a Well-Characterized SARS-CoV-2 Lentiviral Pseudovirus Neutralization Assay Using 293T Cells With Stable Expression of ACE2 and TMPRSS2. PloS One 16, e0248348. doi: 10.1371/ journal.pone.0248348

Pizzato, M., Erlwein, O., Bonsall, D., Kaye, S., Muir, D., and McClure, M. O. (2009). A One-Step SYBR Green I-based Product-Enhanced Reverse Transcriptase Assay for the Quantitation of Retroviruses in Cell Culture Supernatants. J. Virol. Methods 156 (1-2), 1-7 doi: 10.1016/ j.jviromet.2008.10.012

Plescia, C. B., David, E. A., Patra, D., Sengupta, R., Amiar, S., Su, Y., et al. (2020). SARS-CoV-2 Viral Budding and Entry can be Modeled Using BSL-2 Level Virus-Like Particles. J. Biol. Chem. 296, 100103. doi: 10.1074/ jbc.RA120.016148

Schubert, K., Karousis, E. D., Luo, C., Scaiola, A., Echeverria, B., Gurzeler, L. A., et al. (2020). SARS-Cov-2 Nsp1 Binds the Ribosomal mRNA Channel to Inhibit Translation. Nat. Struct. Mol. Biol. 27, 959-966. doi: 10.1038/s41594020-0511-8

Shang, J., Wan, Y., Ye, G., Geng, Q., Auerbach, A., Li, F., et al. (2020). Cell Entry Mechanisms of SARS-Cov-2. Proc. Natl. Acad. Sci. 117, 11727-11734. doi: 10.1073/pnas.2003138117

Sreepadmanabh, M., Sahu, A. K., and Chande, A. (2020). Covid-19: Advances in Diagnostic Tools, Treatment Strategies, and Vaccine Development. J. Biosci. 45, 148. doi: 10.1007/s12038-020-00114-6

Tada, T., Fan, C., Chen, J. S., Kaur, R., Stapleford, K. A., Gristick, H., et al. (2020). An ACE2 Microbody Containing a Single Immunoglobulin Fc Domain Is a Potent Inhibitor of SARS-Cov-2. Cell Rep. 33, 108528. doi: 10.1016/ j.celrep.2020.108528

Tandon, R., Mitra, D., Sharma, P., McCandless, M. G., Stray, S. J., Bates, J. T., et al. (2020). Effective Screening of SARS-CoV-2 Neutralizing Antibodies in Patient Serum Using Lentivirus Particles Pseudotyped With SARS-CoV-2 Spike Glycoprotein. Sci. Rep. 10, 19076. doi: 10.1038/s41598-020-76135-w

Thoms, M., Buschauer, R., Ameismeier, M., Koepke, L., Denk, T., Hirschenberger, M., et al. (2020). Structural Basis for Translational Shutdown and Immune Evasion by 
the Nsp1 Protein of SARS-Cov-2. Science 369, 1249-1255. doi: 10.1126/ science.abc8665

Xia, H., Cao, Z., Xie, X., Zhang, X., Chen, J. Y., Wang, H., et al. (2020). Evasion of Type I Interferon by SARS-Cov-2. Cell Rep. 33, 108234. doi: 10.1016/ j.celrep.2020.108234

Zhang, L., Jackson, C. B., Mou, H., Ojha, A., Peng, H., Quinlan, B. D., et al. (2020). Sars-CoV-2 Spike-Protein D614G Mutation Increases Virion Spike Density and Infectivity. Nat. Commun. 11, 6013. doi: 10.1038/s41467-020-19808-4

Zhang, K., Miorin, L., Makio, T., Dehghan, I., Gao, S., Xie, Y., et al. (2021). Nsp1 Protein of SARS-CoV-2 Disrupts the mRNA Export Machinery to Inhibit Host Gene Expression. Sci. Adv. 7, eabe7386. doi: 10.1126/sciadv.abe7386
Conflict of Interest: The authors declare that the research was conducted in the absence of any commercial or financial relationships that could be construed as a potential conflict of interest.

Copyright (๑) 2021 Mishra, Sreepadmanabh, Ramdas, Sahu, Kumar and Chande. This is an open-access article distributed under the terms of the Creative Commons Attribution License (CC BY). The use, distribution or reproduction in other forums is permitted, provided the original author(s) and the copyright owner(s) are credited and that the original publication in this journal is cited, in accordance with accepted academic practice. No use, distribution or reproduction is permitted which does not comply with these terms. 